\title{
A cytoplasmic long noncoding RNA LINC00470 as a new AKT activator to mediate glioblastoma cell autophagy
}

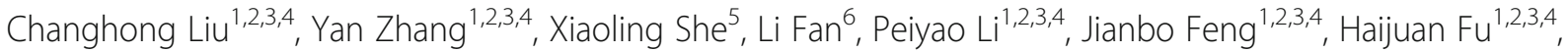

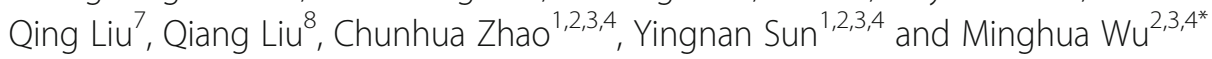

\begin{abstract}
Background: Despite the overwhelming number of investigations on AKT, little is known about IncRNA on AKT regulation, especially in GBM cells.

Methods: RNA-binding protein immunoprecipitation assay (RIP) and RNA pulldown were used to confirm the binding of LINC00470 and fused in sarcoma (FUS). Confocal imaging, co-immunoprecipitation (Co-IP) and GST pulldown assays were used to detect the interaction between FUS and AKT. EdU assay, CCK-8 assay, and intracranial xenograft assays were performed to demonstrate the effect of LINC00470 on the malignant phenotype of GBM cells. RT-qPCR and Western blotting were performed to test the effect of LINC00470 on AKT and pAKT.

Results: In this study, we demonstrated that LINC00470 was a positive regulator for AKT activation in GBM. LINC00470 bound to FUS and AKT to form a ternary complex, anchoring FUS in the cytoplasm to increase AKT activity. Higher PAKT activated by LINC00470 inhibited ubiquitination of HK1, which affected glycolysis, and inhibited cell autophagy. Furthermore, higher LINC00470 expression was associated with GBM tumorigenesis and poor patient prognosis.

Conclusions: Our findings revealed a noncanonical AKT activation signaling pathway, i.e., LINC00470 directly interacts with FUS, serving as an AKT activator to promote GBM progression. LINC00470 has an important referential significance to evaluate the prognosis of patients.
\end{abstract}

Keywords: LncRNA, AKT activation, Oncogene, GBM

\section{Background}

$\mathrm{AKT}$ is a serine/threonine kinase, also known as protein kinase $\mathrm{B}$, which plays critical roles in diverse cellular processes such as proliferation, autophagy, metabolism, and survival [1-4]. Aberrant AKT activation causes a wide variety of disorders including diabetes, neurodegenerative syndromes, and various types of cancers. AKT is well established as the predominant PI3K effector in many cell types [5]. Many cancer genetic alterations deregulate cell signaling pathways and exert their oncogenic effects in part through the PI3K/AKT pathway $[6,7]$.

\footnotetext{
*Correspondence: wuminghua554@aliyun.com

${ }^{2}$ Cancer Research Institute, School of Basic Medical Science, Central South University, Changsha 410078, Hunan, China

${ }^{3}$ Key Laboratory of Carcinogenesis and Cancer Invasion, Ministry of

Education, Changsha 410078, Hunan, China

Full list of author information is available at the end of the article
}

Hence, there is a particularly intimate relationship between the activation of the AKT signaling pathway and tumorigenesis. Activation of PI3K results in the phosphorylation of two key residues on AKT, i.e., Thr308 in the activation motif and Ser473 in a C-terminal hydrophobic motif $[7,8]$. AKT can translocate from the plasma membrane to intracellular compartments, including the cytoplasm and nucleus where it phosphorylates substrates $[9,10]$. Growth factors stimulate phosphorylated AKT to translocate from the cytoplasm to the nucleus [11] where AKT can be phosphorylated and activated [12]. For example, nuclear AKT phosphorylates members of the Foxo subfamily of forkhead transcription factors, promoting nuclear exclusion and thereby inhibiting the transcription of death genes $[13,14]$. Evidence indicates that a number of positive regulators, including regulatory proteins (such

(C) The Author(s). 2018 Open Access This article is distributed under the terms of the Creative Commons Attribution 4.0 International License (http://creativecommons.org/licenses/by/4.0/), which permits unrestricted use, distribution, and 
as PI3K, PTEN, PDK1) [15-17], miRNAs (such as miRNA-7, miRNA-379, and miRNA-126) [18-21], and long noncoding RNAs (lncRNAs, such as LINK-A, lncRNA OIP5-AS1, and MALAT1), promote the overactivation of AKT signaling [22-24]. Until now, the underlying mechanism of AKT in the GBM was not fully understood despite many years of investigation.

Recent studies have revealed the regulatory potential of many lncRNAs involved in numerous physiological and pathological processes [25]. LncRNAs have regulatory roles in gene expression at both transcriptional and post-transcriptional levels in diverse cellular contexts and biological processes [26]. LncRNAs are responsible for nuclear structure integrity and can regulate the expression of either nearby genes (acting in cis in the nucleus) or genes elsewhere in cells (acting in trans in the nucleus or cytoplasm) by interacting with proteins, RNA, and DNA [27-29]. LncRNAs operate through distinct modes, such as signals, scaffolds for protein-protein interactions, molecular decoys, or guides, to target elements in the genome [30,31]. In addition, new types of lncRNAs are likely to be discovered through integrated approaches. For example, sno-lncRNA can form a nuclear accumulation that is enriched in RNA-binding proteins [32].

LINC00470 (also known as C18orf2) is a long non-coding RNA located in chromosome band 18p11.32 between RP11-16P11 and RP11-732L14 [33, 34]. Its alternative splicing of seven exons generates four transcripts. Our previous data demonstrated that LINC00470 expression levels in astrocytoma were significantly higher than those in normal brain tissues [35]. However, the role of LINC00470 remains to be elucidated; in particular, it is not known whether lncRNAs are involved in the regulation of AKT activity in GBM.

In this study, we found that (1) LINC00470 is a positive regulator of AKT activation and it inhibited the nuclear translocation of phosphorylated AKT; (2) LINC00470 directly bound FUS and anchored FUS in the cytoplasm, resulting in FUS activation; (3) LINC00470 interacted with FUS and AKT to form a stable complex; and (4) LINC00470 decreased the ubiquitination of HK1, which affected glycolysis by positively regulating AKT activation in GBM tumorigenesis.

\section{Methods}

\section{Primary tumor cell culture and cell lines}

A primary tumor cell culture was performed as previously described [36]. Astrocytoma cell lines U251 and U87 were bought from cell banks of the Chinese Academy of Sciences (Shanghai, China). All astrocytoma cell lines were subjected to a short tandem repeat (STR) test. U251 and primary tumor cells were cultured in DMEM high-glucose medium with $10 \%$ FBS and a $1 \%$ antibiotic-antimycotic solution (Gibco, Grand Island, NY, USA), while U87 cells were cultured in MEM medium with 10\% FBS and 1\% antibiotic-antimycotic solution at $37^{\circ} \mathrm{C}$ and $5 \% \mathrm{CO}_{2}$.

\section{Antibodies and reagents}

The following primary antibodies were used: AKT (rabbit, Proteintech, 10176-2-AP, WB1:1500, IP:1:250, RIP:1:100); FUS (rabbit, Abcam, ab23439, WB1:2000, IP1: 200, RIP1:100); phospho-Akt (Ser473) (rabbit, Cell Signaling, \#4060, WB1:1500); phospho-Akt (Thr308) (rabbit, Cell Signaling, \#13038, WB1:1500); hexokinase I (rabbit, Cell Signaling, \#2024, WB1:1000); hexokinase II (rabbit, Cell Signaling, \#2867, WB1:1000); Flag (mouse, Sigma-Aldrich, F1804, IP 1:200); GAPDH (mouse, Sangon, D190090, WB 1:5000); H3 (rabbit, Beyotime, AH433, WB 1:500); and p53 (mouse, Active Motif, 39739, WB 1:1000, RIP 1:150). MK-2206 2HCl (S1078) was purchased from Selleck.

\section{LncRNA, siRNAs, and transfection}

Cell transfection was performed using Lipofectamine 3000 (Invitrogen-Life Technologies, Carlsbad, CA, USA) per the manufacturer's instructions.

\section{RNA isolation and RT-qPCR}

This procedure was carried out as previously described. The following primers were used: LINC00470: F: 5' -CGTA AGGTGACGAGGAGCTG-3', R: 5'-GGGGAATGGCTT TTGGGTCA-3'; AKT: F: 5'-GAAGGACGGGAGCAGG C-3', R: 5'-AAGGTGCGTTCGATGACAGT-3'; and GAP DH: F: 5'-AATGGGCAGCCGTTAGGAAA-3', R: 5'-GC GCCCAATACGACCAAATC-3'.

\section{Western blotting}

Details of Western blotting were previously described [37]. Cell lysates were prepared with GLB buffer $(10 \mathrm{mM}$ Tris- $\mathrm{HCl}, \mathrm{pH}=7.5 ; 10 \mathrm{mM} \mathrm{NaCl} ; 0.5 \%$ Triton $\mathrm{X}-100 ; 10 \mathrm{mM}$ EDTA) supplemented with protease inhibitor cocktail (Bimake, Houston, TX, USA, B14001) and phosphatase inhibitor (Bimake, B15001). Cytoplasmic and nuclear proteins were prepared with a Nuclear and Cytoplasmic Protein Extraction Kit (Beyotime, p0028). Thirty-microgram proteins were subjected to electrophoresis in different percentages of gels according to the molecular weight of the detected proteins.

\section{Co-immunoprecipitation assay}

For the interaction of FUS and AKT, HEK293 cells were transfected with the indicated plasmids and extracted by the addition of lysis buffer. For the immunoprecipitation of endogenous FUS and AKT proteins extracted by the addition of lysis buffer, the soluble supernatants were incubated with the indicated antibodies for $1 \mathrm{~h}$ at $4{ }^{\circ} \mathrm{C}$. The immunocomplexes were then precipitated with protein 
A-Sepharose CL-4B. The immunocomplexes were washed three times with lysis buffer, eluted by boiling in sample buffer for SDS-PAGE, and then subjected to immunoblot analysis.

\section{Pulldown assay}

GST fusion proteins containing various deletions of FUS cytoplasmic domain or deletions of AKT were expressed in U251 cells with the pGEX-4T-2 vector and were purified. The lysate was incubated for $1 \mathrm{~h}$ with GST-tagged proteins and glutathione-Sepharose $4 \mathrm{~B}$ beads. The beads were subsequently washed three times in the lysis buffer containing $1 \mathrm{mM}$ EDTA and $0.5 \mathrm{mM}$ DTT. Precipitates were separated by SDS-PAGE and detected by Western blotting analysis.

\section{Cell viability and EdU assays}

This procedure was carried out as previously described [35].

\section{RNA-binding protein immunoprecipitation assay}

Approximately $2 \mu \mathrm{g}$ of the cell extract was mixed with agarose beads, which had already precipitated with the protein antibodies. Beads were washed briefly three times with $\mathrm{GLB}^{+}$lysis, and the retrieved protein was detected by Western blotting. The co-precipitated RNAs were detected by RT-qPCR.

\section{Intracranial implantation mouse model}

All animal experiments were approved by the Animal Care and Use Committee of Central South University. Mouse orthotopic xenograft model was performed as previously described [36]. Six-week SD mice were chosen. Injection of cyclophosphamide once every 2 days. One-centimeter incision was made on the midline, and a 1-mm burr hole was drilled at $\mathrm{AP}=+1 \mathrm{~mm}$ and $\mathrm{MR}=-3 \mathrm{~mm}$ from the bregma at the right hemisphere. Ten microliters of $10^{7}$ cell was infused into the brain at a depth of $-5 \mathrm{~mm}$ from the dura, at a speed of $1 \mu \mathrm{l} / \mathrm{min}$.

\section{Statistical analysis}

All experiments were analyzed with GraphPad Prism 5 (La Jolla, CA, USA). Differences between the different groups were tested using Student's $t$ test or one-way ANOVA. The relationships between the LINC00470 expression and clinic-pathological parameters were examined using the $\chi^{2}$ test. The expression of LINC00470 and patients' survival time was analyzed by single factor and multiplicity factor analysis, and OS curves were calculated using the Kaplan-Meier method with the SPSS 15.0 program (SPSS Inc., Chicago, IL, USA). Data are expressed as the mean \pm S.E.M. from at least three independent experiments. A probability value $P<0.05$ was considered statistically significant.

\section{Results}

LINC00470 was a positive regulator of AKT activities

A vector construct containing the full-length LINC00470 with EGFP tag was developed and assessed for LINC00470 expression. LINC00470 did not have any detectable protein-coding ability (Additional file 1: Figure S1A and B). To investigate biological processes associated with LINC00470 expression in GBM, Pearson correlation analysis between LINC00470 expression and whole genome profiling were performed in GBM samples by TCGA databases. A total of 1802 gene expressions that correlated with LINC00470 expression are shown in Fig. 1a. To investigate which canonical pathways were significantly dysregulated in GBM groups with LINC00470 expression, Fisher's exact test was used to identify 20 canonical pathways in GBM that included PI3K-AKT signaling (Fig. 1a). These analyses indicated that LINC00470 may be associated with PI3K-AKT signaling. Then, we measured the expression levels of LINC00470, AKT, and p-AKT in GBM cell lines and primary cultured GBM cells by RT-qPCR (Additional file 2: Figure S2A) and Western blotting (Additional file 2: Figure S2B). We found a positive correlation between the expression of LINC00470 and p-AKT. In GBM, LINC00470 and AKT had no correlation (Additional file 2: Figure S2B). Overexpression of LINC00470 upregulated the expression of p-AKT ${ }^{\mathrm{T} 308}$ and $\mathrm{p}-\mathrm{AKT}^{\mathrm{S} 473}$ (Fig. 1b). We designed three kinds of siRNA and selected the best interfering effects for follow-up study (Additional file 3: Figure S3), while knockdown LINC00470 reduced $\mathrm{p}-\mathrm{AKT}^{\mathrm{T} 308}$ and p-AKT ${ }^{\mathrm{S} 433}$ levels (Fig. 1c). We also re-expressed LINC00470 in the LINC00470-KD cells. Expression of LINC00470 was elevated after LINC00470 was re-expressed in the LINC00470-KD cells (Fig. 1d upper) and enhanced the $\mathrm{p}-\mathrm{AKT}^{\mathrm{T} 308}$ and $\mathrm{p}-\mathrm{AKT}^{\mathrm{S} 473}$ level (Fig. 1d lower panel). However, neither overexpression nor knockdown of LINC00470 affected total AKT and PI3K expression (Fig. 1b, c and Additional file 4: Figure S4). Therefore, we proposed that LINC00470 modulates AKT activities possibly through a previously unidentified mechanism.

\section{FUS interacted with both LINC00470 and AKT to form a ternary complex in the cytoplasm}

Bioinformatics (http://starbase.sysu.edu.cn/browseRbpLnc RNA.php) predicted FUS, an RNA-binding protein associated with LINC00470, may also bind to AKT. An RIP assay verified the interaction between LINC00470 and FUS (Fig. 2a). A biotin-RNA pulldown assay further confirmed the binding between LINC00470 and FUS (Fig. 2b). Interestingly, RNA-binding protein immunoprecipitation of FUS, but not AKT, specifically retrieved LINC00470 (Fig. 2c). We also performed RIP assay in U87 cells; the result of RIP assay was consistent with that in U251cells (Additional file 5: Figure S5). These results indicated that 


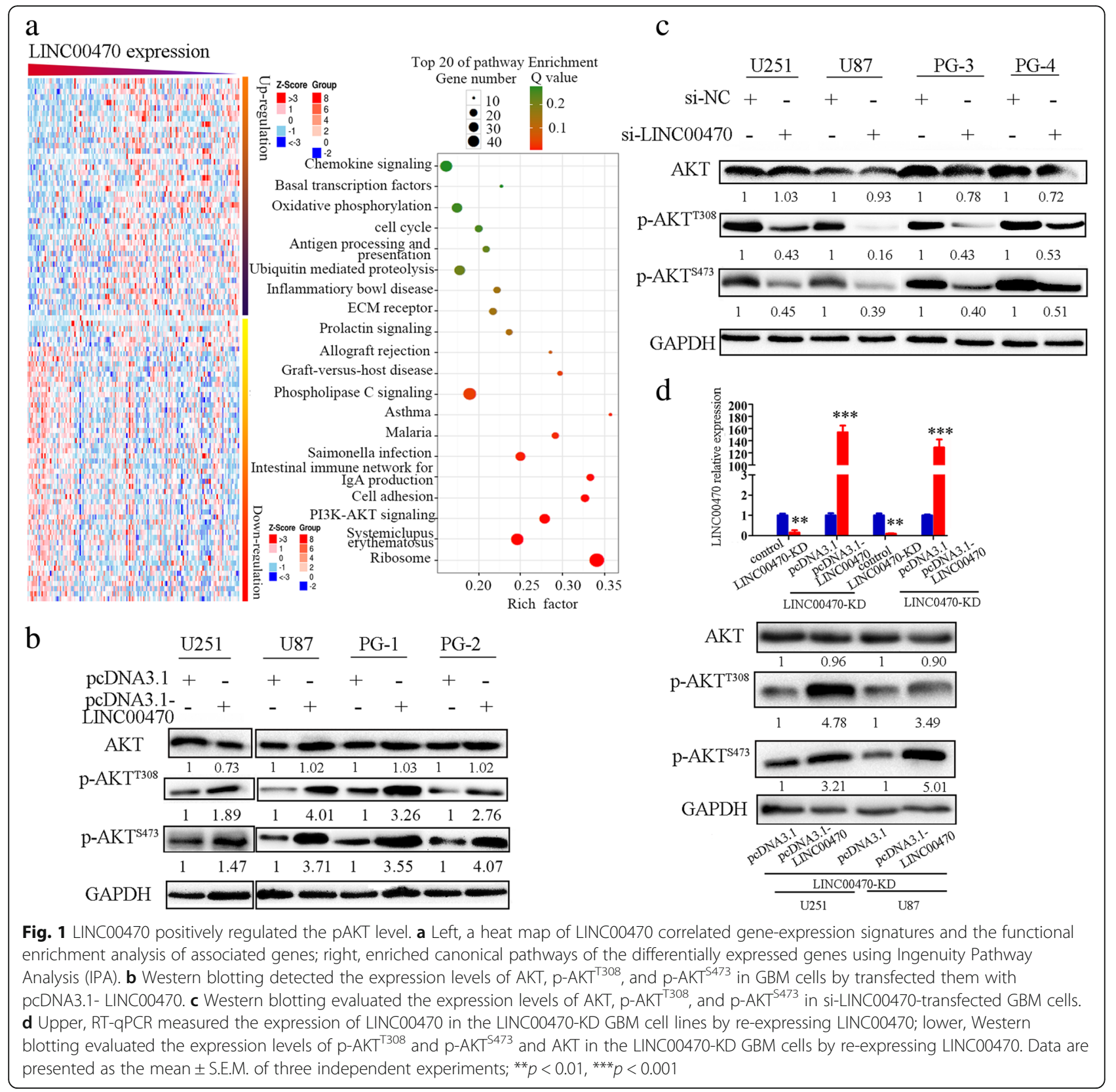

FUS interacted with LINC00470, but there was no direct interaction between LINC00470 and AKT.

To examine the simultaneous existence of LINC00470, FUS, and AKT within the same complex, a two-step Co-IP assay was performed using HEK293 cell lysate from cells in which HA-AKT, Flag-FUS, and pcDNA3.1-LINC00470 were co-transfected. LINC00470 was found in the final immunoprecipitation, suggesting that LINC00470, FUS, and AKT form a ternary complex (Fig. 2d). At the same time, we also found LINC00470, FUS, and AKT can form a ternary complex in U251 cells (Additional file 6: Figure S6). In the absence of LINC00470, FUS and AKT were co-localized in the nucleus of HEK293 cells
(Additional file 7: Figure S7 and Fig. 2e). However, overexpression of LINC00470 anchored FUS and AKT in the cytoplasm (Fig. 2e), while with knockdown of LINC00470 in U251 cells, FUS and AKT translocated from the cytoplasm to the nucleus (Fig. 2f). The interactions between FUS and AKT in the cytoplasm of U251 cells were also verified by Western blotting analysis (Fig. $2 \mathrm{~g}$ left). An RNA pulldown assay showed that the interaction of LINC00470 and AKT disappeared after FUS knockdown in U251 cells (Fig. 2g right). We also found LINC00470 could not affect AKT activation after pcDNA3.1-LINC00470 was transfected into FUS-KD cells (Fig. 2h). The phosphorylated AKT 

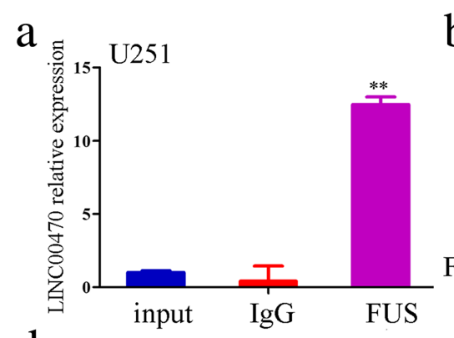

b

$\mathrm{d}$

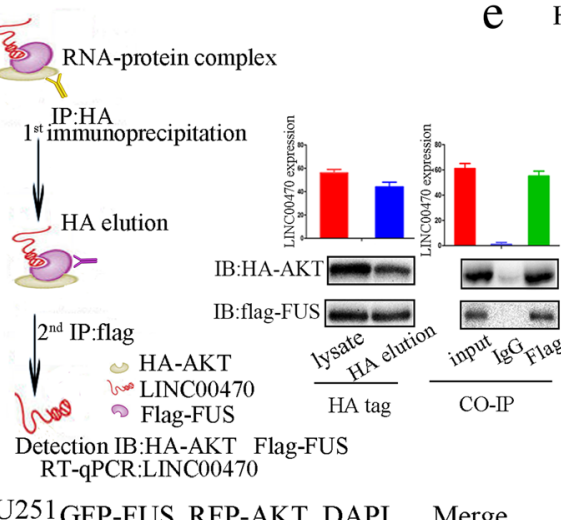

$\mathrm{f}$
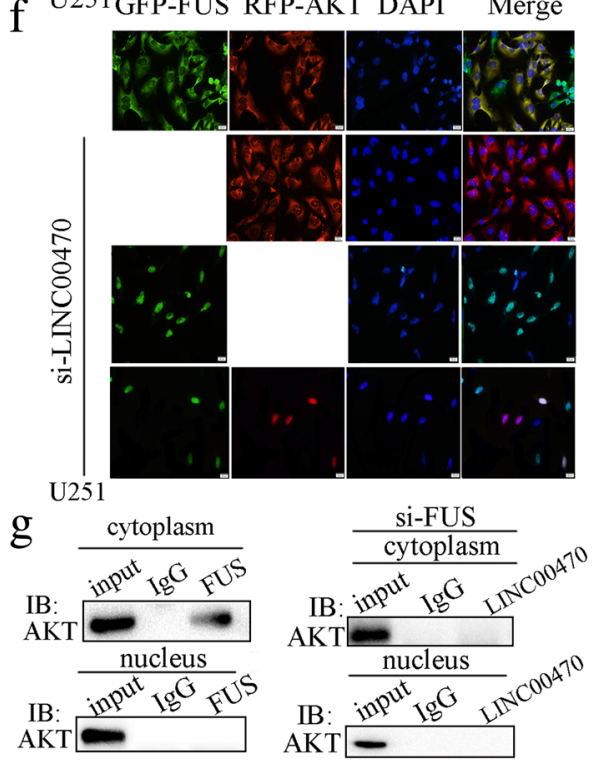

$\mathrm{c}$

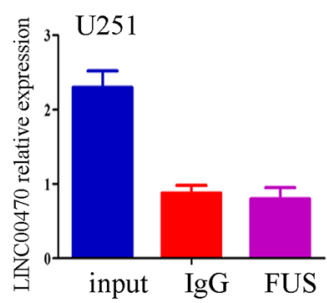

HEK293 GFP-FUS RFP-AKT DAPI Merge

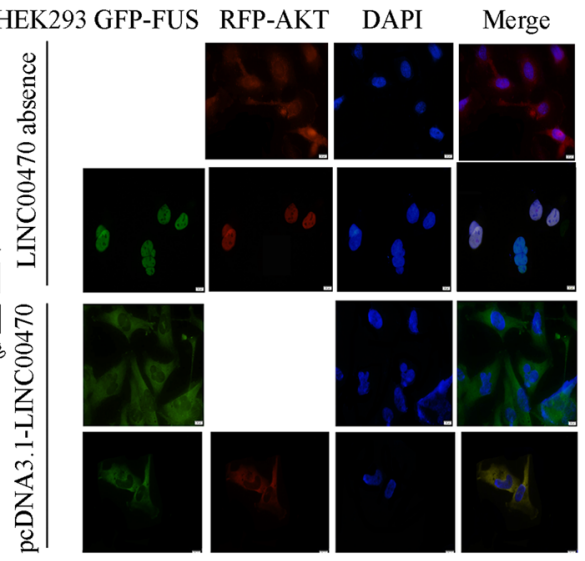

$\mathrm{h}$

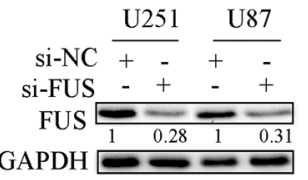

GAPDH $\longrightarrow-0$

pcDNA3.1

si-FUS pcDNA3.1-

LINC00470

AKT

$\mathrm{p}-\mathrm{AKT}^{\mathrm{s}}$

GAPDH

i

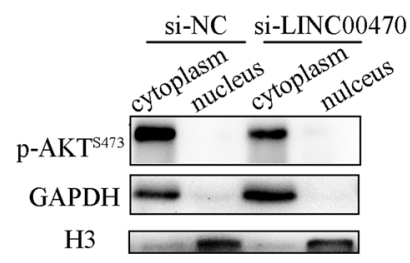

Fig. 2 FUS interacted with LINC00470 and AKT to form a ternary complex in the cytoplasm. a The interaction of LINC00470 and FUS was detected through RIP assays in U251 cells. Data are presented as the mean \pm S.E.M. of three independent experiments. ${ }^{* *} p<0.01$. $\mathbf{b}$ RNA pulldown showed binding between LINC00470 and FUS. c RIP assays showed that there was no interaction between LINC00470 and AKT in U251 cells. Data are presented as the mean \pm S.E.M. of three independent experiments. $\mathbf{d}$ HEK293 cells were transfected with HA-AKT, Flag-FUS, and pcDNA3.1-LINC00470. Two-step co-immunoprecipitation verified their interaction. The expression levels of LINC00470, AKT, and FUS were measured with RT-qPCR and Western blotting, respectively. e The localization of AKT and FUS was detected by immunofluorescence staining in HEK293 cells. $\mathbf{f}$ The co-localization of AKT and FUS was detected by immunofluorescence staining in U251 cells. $\mathbf{g}$ Left, the interactions between endogenous FUS and AKT in the cytoplasm and nucleus were measured by co-immunoprecipitation; right, an RNA pulldown assay showed binding between endogenous LINC00470 and AKT in the cytoplasm and nucleus of U251 cells transfected by si-FUS. $\mathbf{h}$ Western blotting detected the expression of FUS in GBM cells transfected by si-FUS. Expression levels of AKT and p-AKT ${ }^{S 473}$ were measured by Western blotting in GBM cells that re-expressed LINC00470 in FUS-KD GBM cells. i Western blotting detected the expression levels of $\mathrm{p}-\mathrm{AKT}^{\mathrm{S} 473}$ in the cytoplasm and nucleus of U251 cells transfected by si-LINC00470

was reduced in the cytoplasm in LINC00470-knockdown GBM cells (Fig. 2i). The data indicated that LINC00470 promoted the activation of AKT in the cytoplasm by interacting with FUS.

\section{LINC00470 anchored FUS in the cytoplasm and phosphorylated FUS}

Next, a series of LINC00470 deletion mutants were constructed to determine the nucleotides in LINC00470 
that bind to FUS. An RNA pulldown assay showed that there was an interaction between FUS and LINC00470 mutants (1-300 nt, 1-710 nt, 1-1500 nt, 1-2231 nt, and 100-2231 nt), but no interaction between FUS and other LINC00470 deletion mutants (1-100 nt, 300-2231 nt, 710-2231 nt, $1500-2231$ nt, 300-710 nt, 300-1500 nt, and 710-1500 nt) (Fig. 3a), suggesting that the 100-300 nt region of LINC00470 was responsible for its binding to FUS. We also found FUS bound to LINC00470 through its RNA recognition domain (RRM) (Fig. 3b). Confocal fluorescence microscopy indicated that LINC00470 and FUS were mainly co-localized in the cytoplasm in HEK 293 cells after overexpression of LINC00470 (Fig. 3c).

FUS was reported to be continuously shuttling between the nucleus and the cytoplasm [38]. FUS contains multiple post-translational modification sites in the RRM and GGR domain, and post-translational modifications of FUS have profound effects on its binding capacity of DNA, RNA and proteins, changes in protein stability, or subcellular localization $[39,40]$. We speculated that LINC00470 may impact FUS subcellular localization. The nuclear localization of FUS was explored by transient expression of the GFP-FUS fusion plasmid in HEK293 cells (Fig. 3d). When HEK293 cells were co-transfected by pcDNA3.1-LINC00470 and the GFP-FUS fusion plasmid, LINC00470 led to the translocation of FUS from the nucleus to the cytoplasm (Fig. 3d). In GBM cells, the expression of FUS was increased by LINC00470 overexpression and the FUS level was significantly increased in cytoplasm; however, its expression was decreased in the nucleus (Fig. 3e). These data suggested that LINC00470 anchored FUS in the cytoplasm and promoted its expression in the cytoplasm. FUS immunoprecipitation from U251 cells after overexpression of LINC00470 was immunoblotted with anti-phospho- $T$ (threonine phosphorylation) antibodies to assess the level of FUS phosphorylation; LINC00470 promoted phosphorylation of FUS at threonine residues (Fig. 3f). In addition, we also found that LINC00470 was mainly located in the cytoplasm in GBM cells by RNA fluorescence in situ hybridization (Fig. 3g).

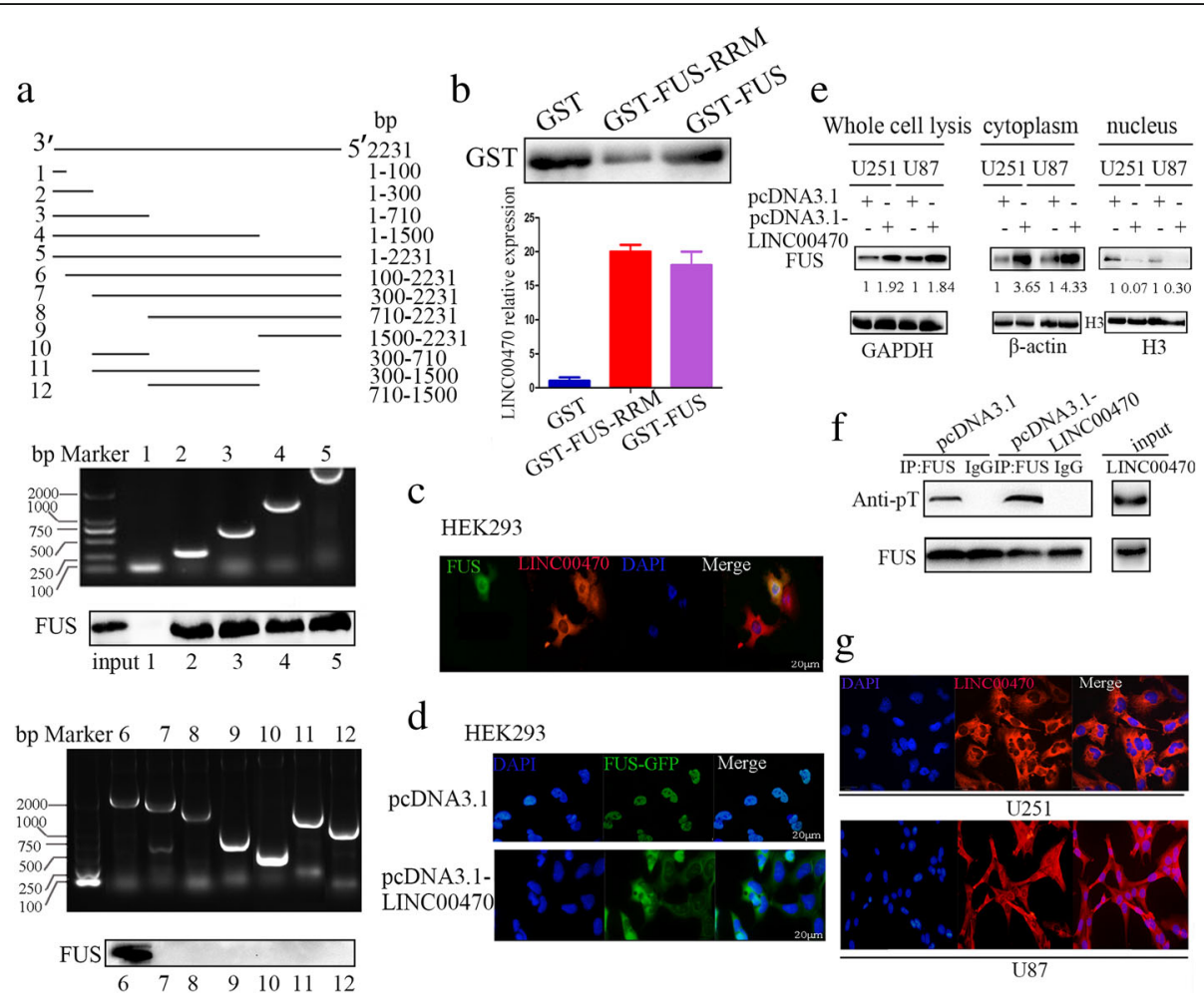

Fig. 3 LINC00470 anchored FUS in the cytoplasm and phosphorylated FUS. a Upper, schematic illustration of substitution mutant constructs of LINC00470; middle and lower, an RNA pulldown assay examined the interaction between FUS and the different mutants of LINC00470. b GST pulldown assays showed that the RRM domain of FUS pulled down LINC00470. c Representative immunofluorescence staining displayed the co-localization of LINC00470 and FUS in the cytoplasm of HEK293 cells after LINC00470 overexpression. Scale bar, $20 \mu \mathrm{m}$. d Representative imaging of LINC00470 anchoring FUS in the cytoplasm in HEK293 cells. Scale bar, $20 \mu \mathrm{m}$. e Western blotting measured the expression of FUS in whole cell lysis and the cytoplasm and nucleus in U251 cells transfected by pcDNA3.1-LINC00470. f Representative immunoprecipitation analysis detected FUS phosphorylation in U251 cells transfected by pcDNA3.1-LINC00470. FUS immunoprecipitated from U251 cells was immunoblotted with pan-phospho-S/TQ antibodies to assess the phosphorylation level of FUS. $\mathbf{g}$ RNA fluorescence in situ hybridization showed the localization of LINC00470 in GBM cells. The nucleus was counterstained with DAPI. Scale bar, $29 \mu \mathrm{m}$ 


\section{FUS bound to AKT and promoted AKT nuclear} translocation and activation

The "Scansite 2.0" software was utilized to identify a docking domain (GGR domain) in FUS, which is an AKT kinase-binding site. GFP-FUS and RFP-AKT expression plasmids were co-transfected into HEK293 cells, CO-IP and immunofluorescence suggested there were interactions between FUS and AKT, and both were co-localized in the nucleus of HEK293 cells (Fig. 4a, b). In addition, we confirmed that endogenous AKT interacted with FUS in the cytoplasm of U251 cells (Fig. 4c, d). Next, a fusion protein of the GGR domain mutation in FUS (GST-FUS-GGR domain) was constructed. A GST pulldown assay indicated that AKT was precipitated with the GST-FUS-GGR peptide (Fig. 4e left) and FUS mainly bound with the $\mathrm{N}$ domain of AKT (Fig. 4e right). Then, we analyzed the changes in AKT protein levels after silencing FUS. Knockdown of FUS did not affect AKT expression. Similarly, FUS expression was not affected by silencing AKT (Fig. 4f). However, we observed that FUS influenced the subcellular localization of AKT by promoting AKT nuclear translocation and increased AKT activation in the nucleus (Fig. 4g).

LINC00470 decreased ubiquitination of HK1 to affect glycolysis by positively regulating AKT activation

$\mathrm{AKT}$, which is frequently dysregulated in cancer, is a well-established regulator of glucose metabolism [41]. a

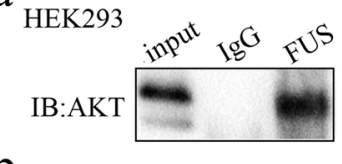

b HEK 293
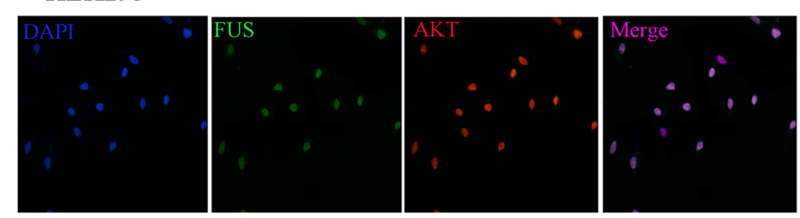

c
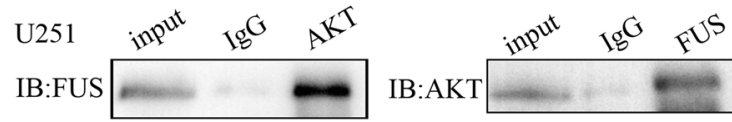

d

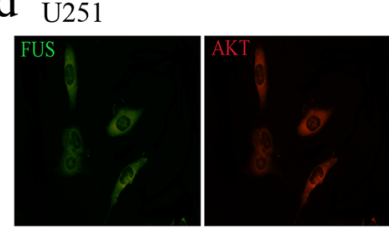

e

GST-FUS

GST G FUS Input
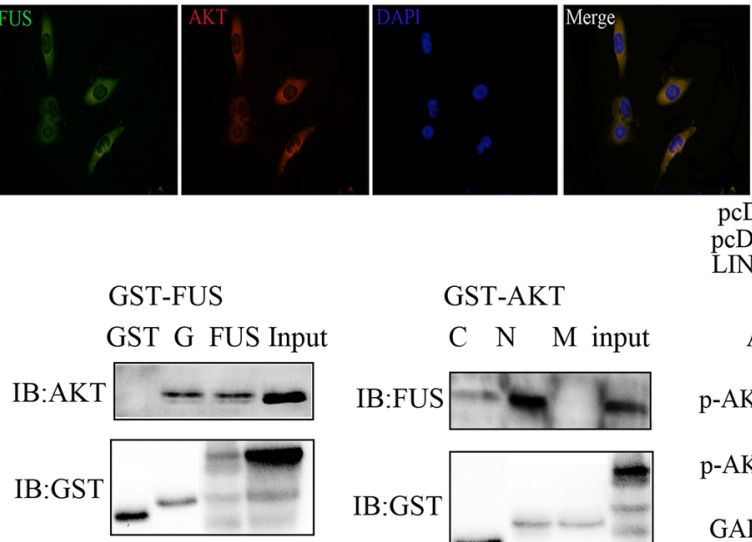

GST-AKT

C $\mathrm{N} M$ input

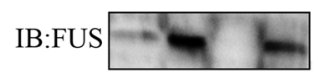

\section{g}

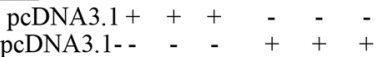
LINC00470

f

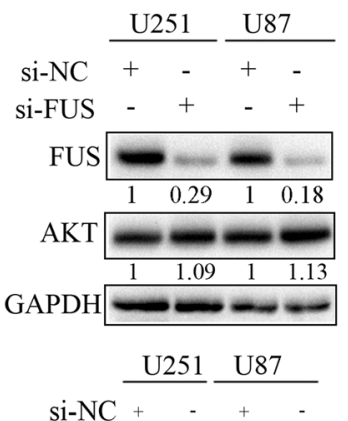

si-AKT

AKT

FUS

GAPDH

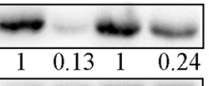

$\begin{array}{lllll}1 & 1.17 & 1 & 0.91\end{array}$

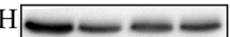

U251

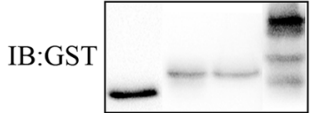

Fig. 4 FUS bound to AKT and promoted AKT activation. a Co-IP analysis measured the exogenous interaction between FUS and AKT in HEK293 cells. b Representative immunofluorescence staining displayed the co-localization of FUS and AKT in the nucleus of HEK293 cells. c Co-IP analysis measured the endogenous interaction between FUS and AKT in U251 cells. d Representative immunofluorescence staining displayed the endogenous co-localization of FUS and AKT in the cytoplasm of U251 cells. e Left, GST pulldown assays showed that the GGR domain of FUS pulled down AKT; right, GST pulldown assays showed that the N-terminal region of AKT mainly pulled down FUS. $\mathbf{f}$ Upper, Western blotting measured the expression levels of FUS and AKT in GBM cells transfected by si-FUS; lower, Western blotting measured the expression levels of AKT and FUS in GBM cells transfected with si-AKT. $\mathbf{g}$ Western blotting measured the expression levels of AKT and pAKT in the whole lysis, cytoplasm, and nucleus of U251 cells transfected by pcDNA3.1-FUS 
Its regulation on metabolic processes is required for tumor proliferation, apoptosis, and autophagy [42-44]. Enforcing or silencing LINC00470 expression in GBM cells increased or reduced glycolysis uptake and lactate production, respectively (Fig. 5a, b). Hexokinases catalyze the first and irreversible step of glucose metabolism, i.e., the ATP-dependent phosphorylation of glucose to yield glucose-6-phosphate [45]. Overexpression of LINC00470 increased the total hexokinase activity in U251 cells compared to controls, and HK activity was inhibited after knockdown of LINC00470 in U251 cells (Fig. 5c). HK1 is a major isoform of HK and is the first key enzyme in the glycolysis pathway [45]. Importantly, the protein expression level of HK1 was markedly increased in response to LINC00470 overexpression (Fig. 5d). In contrast, we found that $\mathrm{HK} 2$, another major isoform of HK, was not changed statistically significantly in LINC00470-overexpressed cells (Fig. 5d).

Next, we explored the molecular mechanisms underlying the LINC00470 that affects the activity of HK1. Inhibiting the activity of AKT with MK-2206 resulted in downregulated of HK1 (Fig. 5e). Additionally, we transfected both pcDNA3.1 and pcDNA3.1-LINC00470 vectors into U251
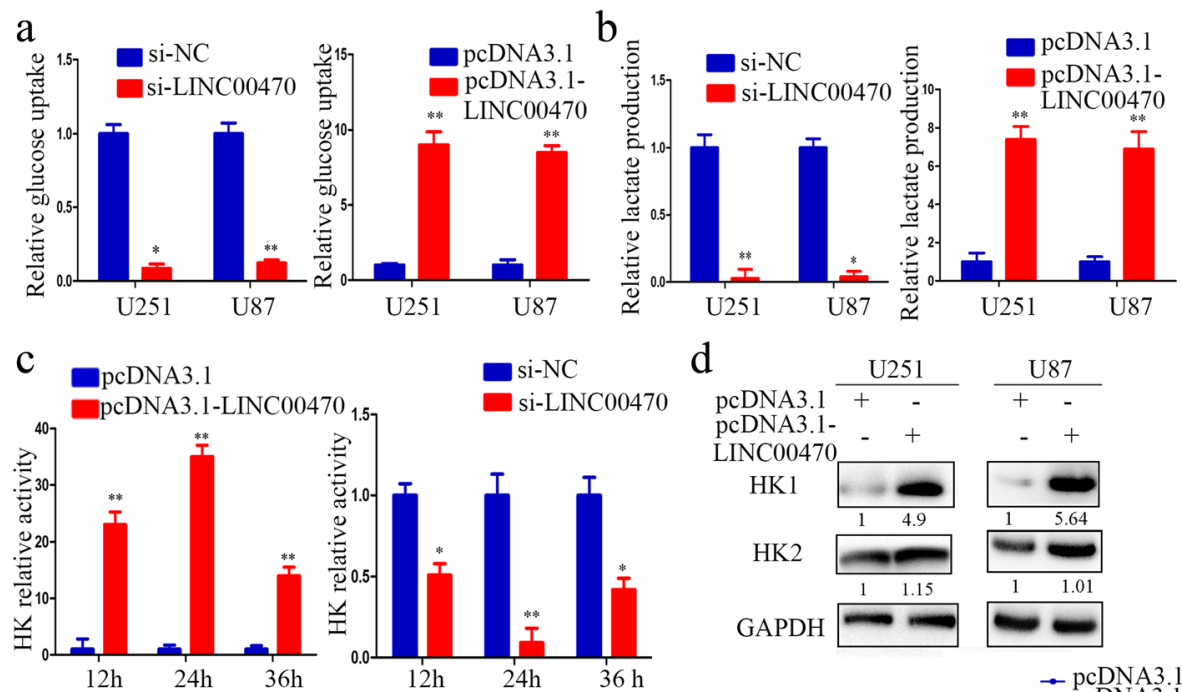

d
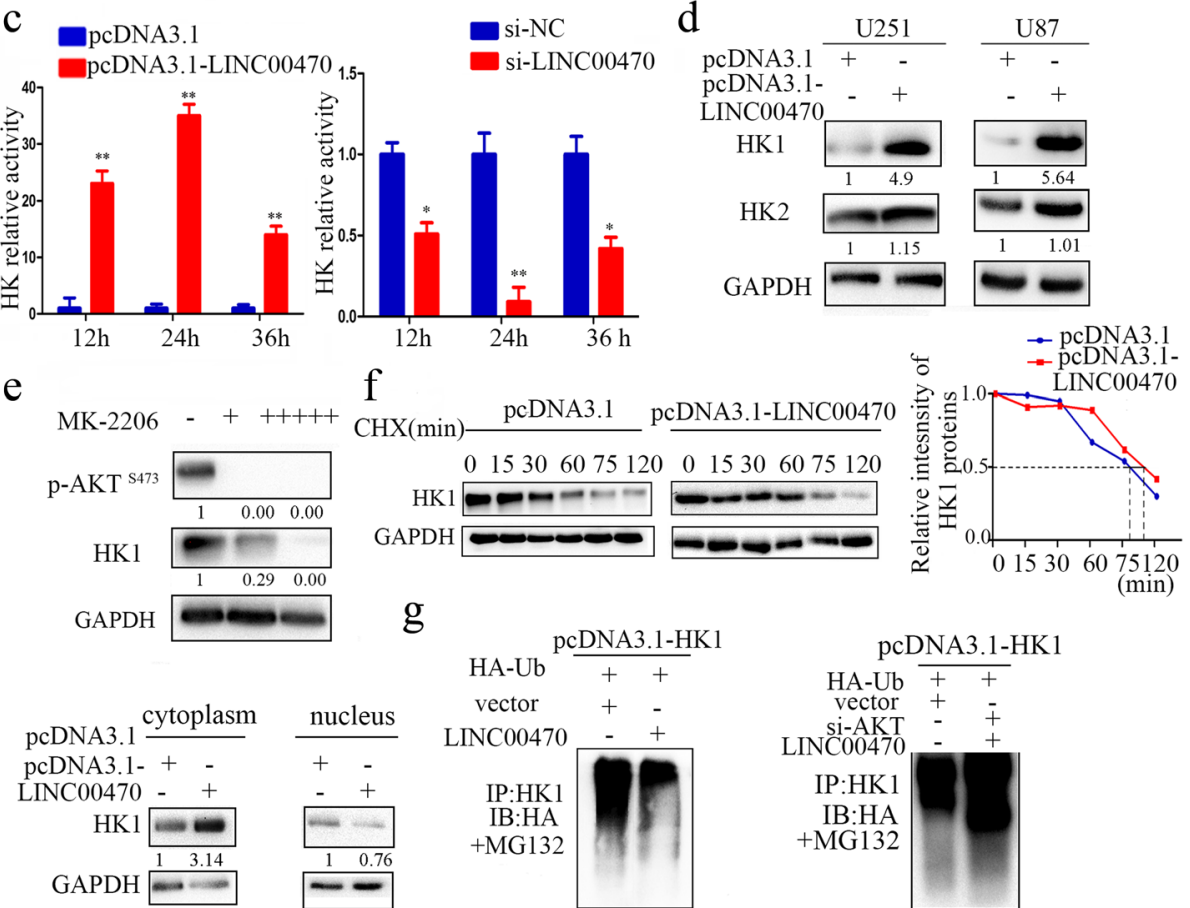

g
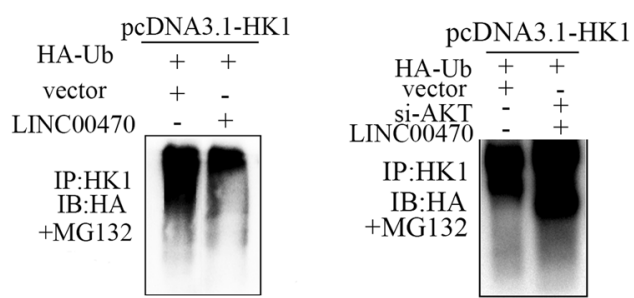

Fig. 5 LINC00470 inhibited HK1 ubiquitination to affect glycolysis by positively regulating AKT activation. a RT-qPCR measured the expression of LINC00470 in the GBM cell lines; GBM cells were transfected with si-LINC00470 or pcDNA3.1-LINC00470. Data are presented as the mean \pm S.E.M. of three independent experiments; ${ }^{*} p<0.05,{ }^{* *} p<0.01$. b Relative levels of glucose uptake and lactate production were detected in GBM cells. GBM cells were transfected with si-LINC00470 or pcDNA3.1-LINC00470. Data are presented as the mean \pm S.E.M. of three independent experiments; ${ }^{*} p<0.05,{ }^{* *} p<0.01$. c HK activity was measured at different time points after GBM cells were transfected with pCDNA3.1-LINC00470 or si-LINC00470. Data are presented as the mean \pm S.E.M. of three independent experiments; ${ }^{* *} p<0.01,{ }^{* * *} p<0.001$. $\mathbf{d}$ Western blotting detected the expression levels of HK1 and HK2 in GBM cells transfected by LINC00470. e Upper, Western blotting detected the expression levels of p-AKT ${ }^{S 473}$ and HK1 in U251 cells. The cells were treated with different concentrations of AKT inhibitor (MK-2206; +, $\left.1 \mu \mathrm{M} ;++++++, 5 \mu \mathrm{M}\right)$; lower, Western blotting detected the expression levels of HK1 in the cytoplasm and nucleus in U251 cells transfected by pcDNA3.1-LINC00470. f The half-life of HK1 was assessed in U251 cells. Cells were transfected with pcDNA3.1-LINC00470. $\mathbf{g}$ The relative amount of ubiquitination HK1 was determined by a ubiquitination assay in U251 cells transfected by LINC00470 or si-AKT and LINC00470 
cells, then analyzed the protein levels of HK1 in the cytoplasm and nucleus. HK1 expression increased in the cytoplasm under different experimental conditions; concomitantly, HK1 expression in the nucleus did not change significantly (Fig. 5e). To determine how HK1 protein changed, we treated U251 cells with cycloheximide (CHX) and analyzed the stability of HK1 in response to LINC00470 overexpression. The half-life of

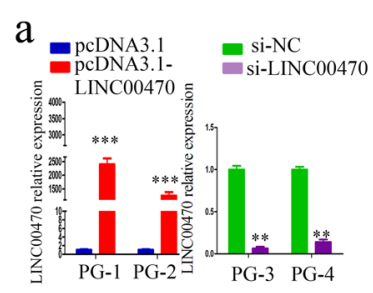

C $\quad \underline{P G-1}$ $\underset{\text { pcDNA3.1 }}{\text { pcDNA3.1- }}+$ LINC00470

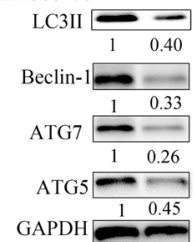

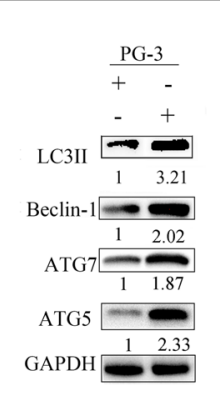

d

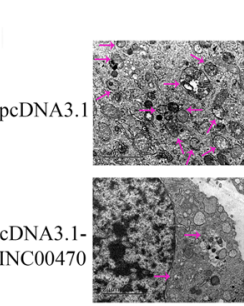

b

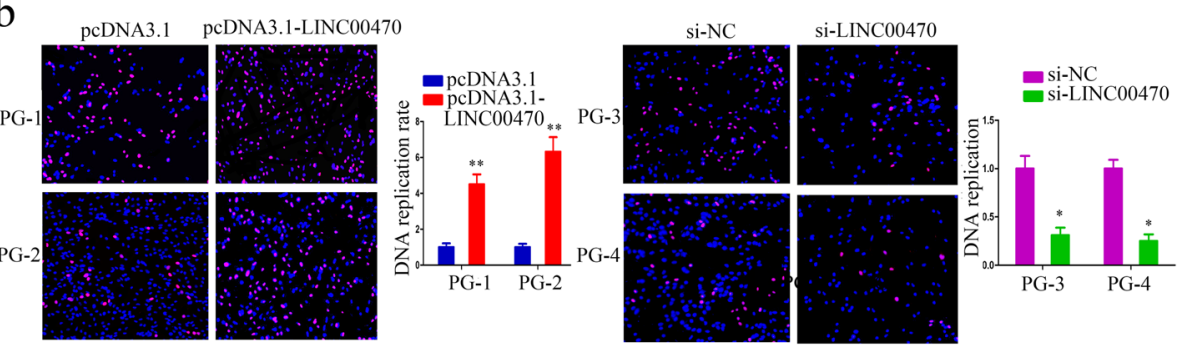

e

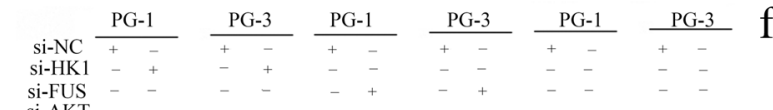
si-FUS pcDNA3 3 .

pcDNA3.1-
LINC00470
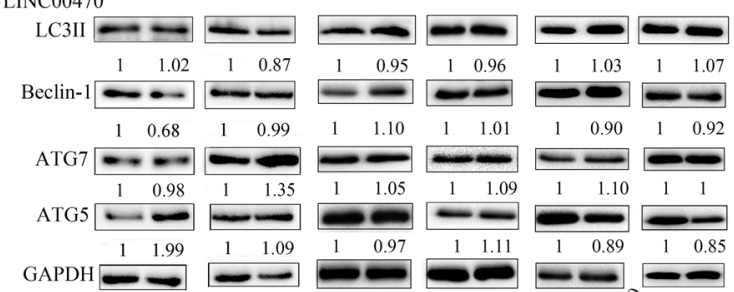

$-$

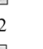

(1)
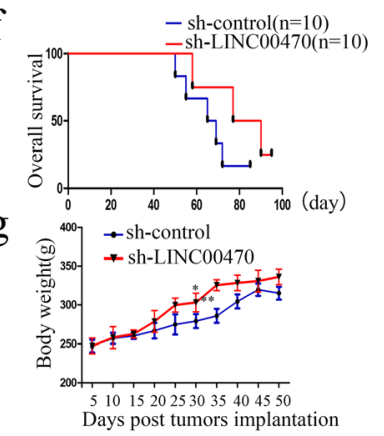

$\mathrm{h}$

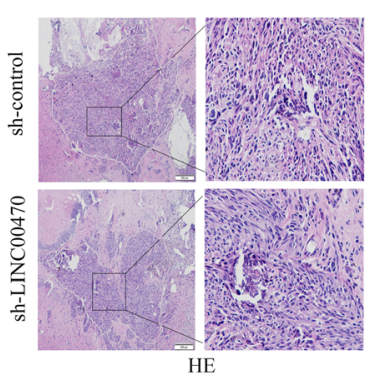

i
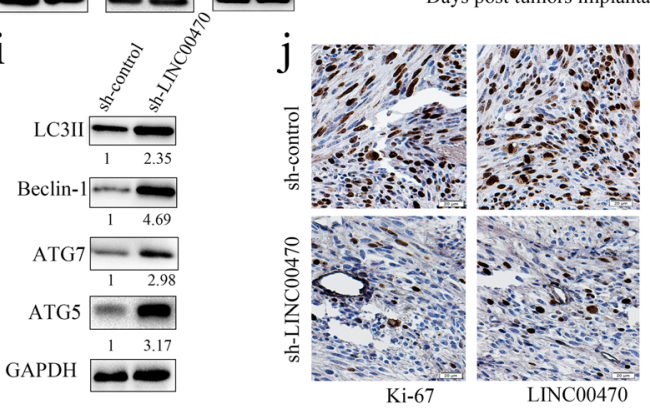

Fig. 6 LINC00470 promoted the tumorigenesis of GBM cells. a Expression levels of LINC00470 were measured by RT-qPCR in primary cultured GBM cells (LINC00470 had relatively low expression in PG-1 and PG-2; LINC00470 had relatively high expression in PG-3 and PG-4). Primary cultured GBM cells were transfected with si-LINC00470 or pcDNA3.1-LINC00470. Data are presented as the mean \pm S.E.M. of three independent experiments; ${ }^{* *} p<0.01,{ }^{* * *} p<0.001$. b An EDU assay was applied to assess cell proliferation of primary cultured GBM cells. Primary cultured GBM cells were transfected with pcDNA3.1LINC00470 or si-LINC00470. c Western blotting measured the expression levels of autophagy marker LC3, beclin-1, ATG7, and ATG5 in PG-1 and PG-3 cells. The cells were transfected with pcDNA3.1-LINC00470 or si-LINC00470. d Electron microscopy detected the autophagy of U251 cells transfected with pcDNA3.1-LINC00470. e Western blotting measured the expression levels of autophagy marker LC3, beclin-1, ATG7, and ATG5 in PG-1 and PG-3 cells. The cells were transfected with si-HK1, si-FUS or si-AKT. f Survival analysis showed that Sprague Dawley rats transplanted with U251-sh-LINC00470 cells have longer overall survival. g Tumor growth for U251-sh-control and U251-sh-LINC00470 in Sprague Dawley rats.* $p<0.05,{ }^{* *} p<0.01$. h H\&E staining showed the volume and morphology of tumors in mice transplanted with U251-sh-LINC00470 cells. The white circle represents the size of the tumor. $\mathbf{i}$ Western blotting measured the expression levels of the autophagy marker LC3, beclin-1, ATG7, and ATG5 in intracranial transplanted tumors. j Expression of Ki-67 and LINC00470 in intracranial transplanted tumors was detected by immunohistochemical staining or in situ hybridization, respectively 
HK1 was much longer in LINC00470-overexpressed cells than that in controls (Fig. 5f). We further explored the mechanism of AKT-mediated HK1 regulation and found lower HK1 ubiquitination levels in LINC00470-transfected cells treated with MG132, and a restoration experiment was performed by knocking down AKT. We found that the ubiquitination level of HK1 was rescued (Fig. 5g). Together, these observations suggested that LINC00470 affected the ubiquitination and expression of HK1 through activating AKT.

\section{LINC00470 is an onco-RNA, and it induced the malignant characteristics of GBM cells}

The above data suggested that LINC00470 plays an important role in GBMs. Accordingly, the primary cultured cells were used to evaluate the functions of LINC00470. As shown in Additional file 7: Figure S7, there was relatively low expression of LINC00470 in PG-1 and PG-2 cells and relatively high expression of LINC00470 in PG-3 and PG-4 cells. Therefore, we expected overexpression of LINC00470 in PG-1 and PG-2 cells and knockdown of LINC00470 in PG-3 and PG-4 cells (Fig. 6a). We found overexpression of LINC00470 contributed to the proliferation of PG1 and PG2 cells by CCK-8 assay (Fig. 6b and Additional file 8: Figure S8). Knockdown of LINC00470 in PG-3 and PG-4 cells decreased cell proliferation (Fig. 6b and Additional file 8: Figure S8). Autophagy primarily promoted the progression of cancers $[46,47]$. We also found that, in PG-1 cells, overexpression of LINC00470 inhibited the levels of autophagy (Fig. 6c, d), and in PG-3 cells, knockdown of LINC00470 promoted the levels of autophagy (Fig. 6c).

To evaluate whether glycolysis activation serves as an upstream mechanism for LINC00470-mediated autophagy, we monitored the markers of autophagy by knockdown of HK1, FUS, and AKT, respectively. The

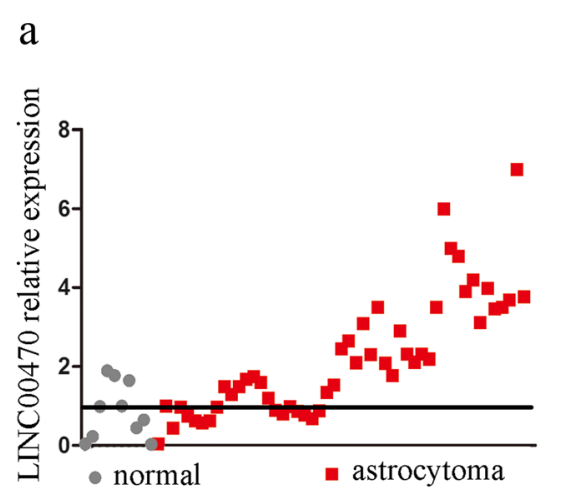

C
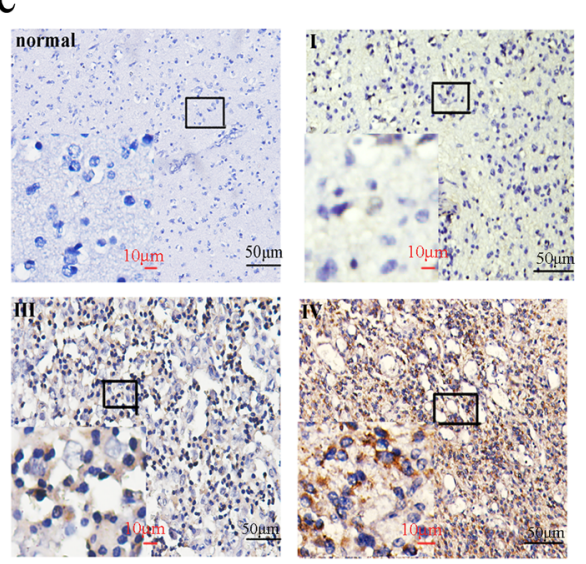

b

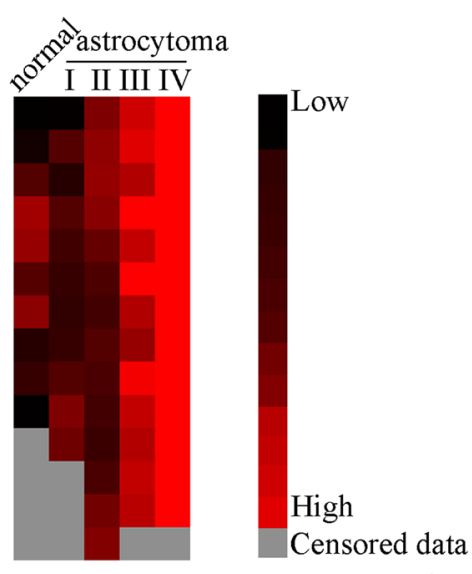

$\mathrm{d}$

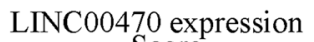

High $S_{6-7}^{\text {Score }}$

Low $-\begin{aligned} & 3-5 \\ & 1-2\end{aligned}$
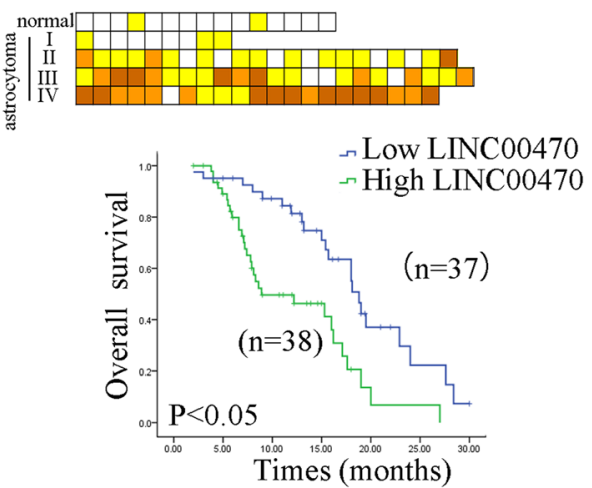

Fig. 7 LINC00470 was an independent prognostic factor in astrocytoma patients. a RT-qPCR detected the expression levels of LINC00470 in normal brain tissues and astrocytoma. b RT-qPCR measured the expression levels of LINCO0470 in astrocytoma with different WHO grades of astrocytoma. c The expression levels of LINC00470 were detected in astrocytoma tissues via in situ hybridization. Black scale bars, 50 um; red scale bars, $10 \mu \mathrm{m}$. d Upper, the score of in situ hybridization in astrocytoma tissues; lower: Kaplan-Meier analysis for overall survival in 75 astrocytomas in high- and low-risk groups based on LINC00470 expression levels 
results showed that when HK1, FUS, or LINC00470 was knocked down, the autophagy level of GBM did not decrease. These results suggested that LINC00470 affected autophagy that was required for HK1, FUS, and AKT (Fig. 6e). At the same time, we applied an intracranial orthotopic transplanted model to evaluate whether LINC00470 mediated GBM tumorigenesis. Compared to mice transplanted with U251-sh-control cells, mice transplanted with U251-sh-LINC00470 cells exhibited longer survival (Fig. 6f), gained more weight (Fig. 6g), and had smaller tumors (Fig. 6h). Knockdown of LINC00470 significantly increased the autophagy levels and decreased the expression of Ki-67 and LINC00470 in an intracranial orthotopic transplanted model (Fig. 6i, j).

\section{LINC00470 was an independent prognostic factor in astrocytoma patients}

To further evaluate the clinical significance of LINC00470 in astrocytomas, including GBMs, we found that the levels of LINC00470 were significantly increased in astrocytoma tissues $(n=60)$ compared with normal brain tissues $(n=12)$ by RT-qPCR (Fig. 7a), especially in high-grade astrocytomas (Fig. 7b). We next measured LINC00470 levels in a panel of 75 astrocytoma tissues and 15 normal brain tissues by in situ hybridization (Fig. 7c). The results were consistent with those of RT-qPCR (Fig. 7a).

Subsequently, we conducted a univariate cox regression analysis using clinical variables for astrocytoma patients and found that expression of LINC00470, astrocytoma grade, patients' age, and the astrocytoma location were statistically associated with overall survival (Table 1). The multivariate cox proportional hazards model indicated that LINC00470 expression and astrocytoma grades were independently associated with overall survival (hazard ratio $[\mathrm{HR}]=2.876, P=0.02 ; \mathrm{HR}=1.892$, $P=0.044$; respectively) (Table 2 ). The results showed that LINC00470 was an independent prognostic factor in astrocytoma patients.

The patients were divided into high or low LINC00470 expression groups according to the ISH scores. Kaplan-Meier analysis of the 75 patients with astrocytoma revealed that high LINC00470 expression levels significantly correlated with shorter survival times (Fig. 7d). High LINC00470 expression was significantly associated with a poor prognosis of astrocytoma patients.

\section{Discussion}

Previous studies have shown multiple signaling pathways that are misregulated in human glioblastomas, such as RTK/PI3K/AKT/Foxos signaling pathway, p53, and Rb1 tumor suppressor pathways [48]. Given the
Table 1 Correlation between the clinicopathological factors and expression of LINC00470 in astrocytoma

\begin{tabular}{|c|c|c|c|}
\hline Characteristic & Total $(\mathrm{N}=75)$ & $\begin{array}{l}\text { LINC00470 high } \\
\text { expression }\end{array}$ & $\begin{array}{l}\text { LINC00470 low } \\
\text { expression }\end{array}$ \\
\hline \multicolumn{4}{|l|}{ Histologic grade*-no.(\%) } \\
\hline \multicolumn{4}{|l|}{ Astrocytoma } \\
\hline । & $9(12)$ & $3(33)$ & $6(67)$ \\
\hline ॥ & $27(36)$ & $14(52)$ & $13(48)$ \\
\hline III & $18(24)$ & $13(72)$ & $15(28)$ \\
\hline IV & $21(28)$ & $17(81)$ & $4(19)$ \\
\hline \multicolumn{4}{|l|}{ Sex-no.(\%) } \\
\hline Male & $39(52)$ & $17(44)$ & $22(56)$ \\
\hline Female & $36(48)$ & $21(58)$ & $15(42)$ \\
\hline \multicolumn{4}{|l|}{ Age ${ }^{*}$ no.(\%) } \\
\hline$\leq 42$ & $31(41)$ & 10(33) & $21(67)$ \\
\hline$>42$ & $44(54)$ & $35(80)$ & $9(20)$ \\
\hline \multicolumn{4}{|c|}{ Tumor location"-no./total no.(\%) } \\
\hline Frontal lobe & 23/72(32) & 9/23(39) & $14 / 23(61)$ \\
\hline Parietal lobe & 19/72(26) & $6 / 19(32)$ & 13/19(68) \\
\hline Temporal lobe & 13/72(19) & 10/13(77) & $3 / 13(23)$ \\
\hline Brainstem & $3 / 72(4)$ & $2 / 3(66)$ & $1 / 3(34)$ \\
\hline others & 14/72(19) & $7 / 17(50)$ & $7 / 17(50)$ \\
\hline \multicolumn{4}{|l|}{ Laterality-no./total no.(\%) } \\
\hline Left & $32 / 72(44)$ & 15/33(47) & 17/33(53) \\
\hline Right & 25/72(35) & 10/26(38) & $16 / 26(62)$ \\
\hline others & $15 / 72(21)$ & $7 / 16(43)$ & $9 / 16(57)$ \\
\hline \multicolumn{4}{|c|}{ Presenting symptom-no./total no.(\%) } \\
\hline Seizure & $38 / 70(54)$ & $21 / 38(55)$ & $17 / 38(45)$ \\
\hline Headache & $12 / 70(17)$ & $6 / 12(50)$ & $6 / 12(50)$ \\
\hline $\begin{array}{l}\text { Sensory or visual } \\
\text { change }\end{array}$ & $9 / 70(13)$ & $5 / 9(56)$ & $4 / 9(44)$ \\
\hline Mental statue change & 11/70(16) & $6 / 11(55)$ & $5 / 11(45)$ \\
\hline
\end{tabular}

Categorical distributions were compared with the use of Fisher's exact test. ${ }^{*} \mathrm{P}<0.01$ for the difference among the molecular subtypes.

complexity and redundancy of the signaling networks associated with glioma, targeting of critical oncogenic pathways might constitute a promising treatment approach [49]. For example, S109 treatment disturbed three pathways in glioma including the RTK/AKT/ Foxos signaling pathway and the p53 and $\mathrm{Rb} 1$ tumor-suppressor pathways [48]. Although a multitude of studies have demonstrated the importance of PI3K in the activation of AKT, there have been reports suggesting that AKT activation can proceed in a manner that is independent of PI3K [2]. In the present study, we provided the evidence that LINC00470 was required for AKT cytoplasm activation and the interaction of LINC00470 and FUS was 
Table 2 Summary of multivariate analysis of Cox proportional hazards model for survival of patients with astrocytoma

\begin{tabular}{|c|c|c|c|c|}
\hline \multirow[t]{2}{*}{ Variable } & \multicolumn{2}{|c|}{$\begin{array}{l}\text { Univarible } \\
\text { Regression }\end{array}$} & \multicolumn{2}{|c|}{$\begin{array}{l}\text { Multivariable } \\
\text { Regression }\end{array}$} \\
\hline & $\mathrm{HR}$ & $P$ & $\mathrm{HR}$ & $\mathrm{P}$ \\
\hline Gender(Female vs. Male) & 1.23 & 0.244 & 1.150 & 0.593 \\
\hline Age & 1.46 & 0.332 & 1.398 & 0.475 \\
\hline \multicolumn{5}{|l|}{ Grade } \\
\hline I+II vs. III + IV & 1.741 & 0.051 & 1.892 & 0.044 \\
\hline Tumor location & 1.021 & 0.871 & 1.566 & 0.111 \\
\hline Laterality & 1.381 & 0.211 & 1.522 & 0.169 \\
\hline Presenting symptom & 0.721 & 0.879 & 0.901 & 0.351 \\
\hline HighLINC00470 expression & 2.113 & 0.030 & 2.876 & 0.021 \\
\hline
\end{tabular}

critical for AKT activation. Our results provided a new mechanism for AKT activity regulation, and we uncovered noncanonical AKT activation signaling by long non-coding RNA.

Recently, the study of lncRNAs has become important, with emerging evidence indicating that lncRNAs function as oncogenes and tumor suppressors, thus having an impact on one or more of the cancer hallmarks $[50,51]$. The roles of a small number of lncRNAs such as HOTAIR, H19, and MALAT1 have been depicted in cancers, but little is known about LINC00470. Our study suggested an oncogenic role for LINC00470 in GBM. This was based on the following lines of evidence: (1) LINC00470 was upregulated in GBM and its expression was positively correlated with p-AKT; (2) ectopic expression of LINC00470 or knockdown of LINC00470 increased or suppressed AKT activity and tumor cell proliferation, respectively; and (3) re-expression

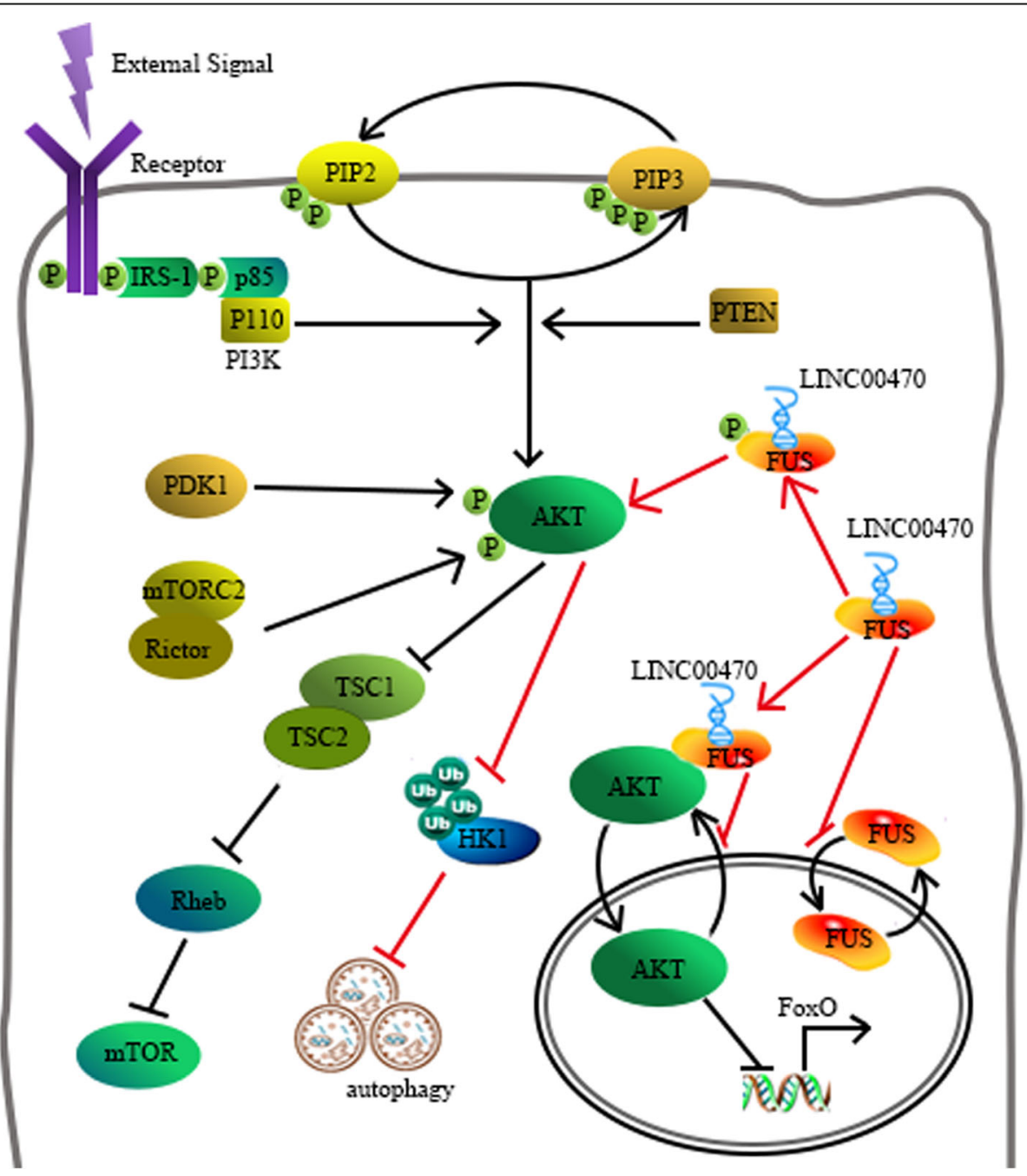

Fig. 8 A schematic diagram of the working model for the LINC00470 targeting system in GBM cells 
of LINC00470 in LINC00470-KO cells was able to restore AKT activation.

FUS is a member of the Ewing's sarcoma family of proteins that appears to translocate from the cytoplasm to the nucleus [52], and it is phosphorylated in response to radiotherapy [53]. However, to date, there is no evidence that lncRNAs can regulate FUS localization and activation. Our study demonstrated that FUS was a new binding partner of LINC00470. LINC00470 bound FUS, anchored it in the cytoplasm, and increased FUS expression in the cytoplasm to activate it. Our results not only revealed that FUS could be used as molecular scaffolding that bound LINC00470 and AKT but also upregulated phosphorylated AKT. In HEK293 cells with the absence of LINC00470, FUS was mainly located in the nucleus, and it transported AKT to the nucleus. However, in GBM cells, LINC00470 prevented FUS from transporting to the nucleus, so AKT was also activated and anchored in the cytoplasm. High levels of p-AKT decreased ubiquitination of $\mathrm{HK} 1$, so that the HK1 protein degradation rate was inhibited, and a higher level of HK1 affected glycolysis and inhibited cell autophagy. Our results further suggested that LINC00470 mediated AKT activation, at least in part, through interaction with FUS.

Finally, we confirmed the prognostic value of LINC00470 and that the high level expression of LINC00470 was an unfavorable prognosis marker for astrocytoma patients. Patients with high expression of LINC00470 had shorter survival times than those with low expression of LINC00470.

\section{Conclusions}

To summarize, we first demonstrated the function of LINC00470 in GBM and manifested a new regulatory mechanism for AKT activation. These results will provide a theoretical and experimental basis for verifying the mechanism of GBM carcinogenesis and identifying biomarkers for the early diagnosis and prognosis in GBM (Fig. 8).

\section{Additional files}

Additional file 1: Bioinformatics analyses of evolutional conservation and protein-coding potential of LINC00470. A: the analysis of protein coding potential of LINC00470 using tools provided by the Peking University Center for Bioinformatics (cpc.cbi.pku.edu.cn/programs/run_cpc.jsp) shows LINC00470 lack of protein-coding capability. B: plasmids as schematically shown at left were transfected to HEK293 cells (right). Immunoblotting using antibody specific to ERK and fluorescent imaging showed that LINC0040-EGFP plasmid did not express GFP. (DOCX 755 kb)

Additional file 2: The relationship between LINC00470, AKT, and p-AKT. A: RT-qPCR and Western blotting measured the expression of LINC00470 and AKT in GBM cell lines and primary GBM cells. Data presented as mean \pm S.E.M. of three independent experiments. B: Western blotting measured the expression of AKT and P-AKT in GBM cell lines and primary
GBM cells. Data showed positive correlation between the expression of LINC00470 and p-AKT in GBM. (DOCX 302 kb)

Additional file 3: Effect of LINC00470 knockdown in GBM cells. RT-qPCR measured the expression of LINC00470 in GBM cell lines and primary GBM cells. Data presented as mean \pm S.E.M. of three independent experiments. (DOCX $168 \mathrm{~kb}$ )

Additional file 4: The expression of PI3K in GBM cells. The expression of PI3K was measured by Western blotting in GBM cells. (DOCX 204 kb)

Additional file 5: The associate between LINC00470, FUS, and AKT in U87 cells. A: the interaction of LINC00470 and FUS was detected through RIP assays in U87 cells. Data are presented as the mean \pm S.E.M. of three independent experiments. ${ }^{* *} p<0.01$. B: RNA pulldown showed binding between LINC00470 and FUS. Data are presented as the mean \pm S.E.M. of three independent experiments. C: RIP assays showed that there was no interaction between LINC00470 and AKT in U87 cells. Data are presented as the mean \pm S.E.M. of three independent experiments. (DOCX 264 kb)

Additional file 6: LINC00470, FUS, and AKT can form a ternary complex in U251 cells. The expression levels of LINC00470, AKT, and FUS were measured by RT-qPCR and Western blotting, respectively. Data presented as mean \pm S.E.M. of three independent experiments. (DOCX $1262 \mathrm{~kb}$ )

Additional file 7: The expression levels of LINC00470 in GBM cells. The expression levels of LINC00470 were measured by RT-qPCR. Data presented as mean \pm S.E.M. of three independent experiments. (DOCX $129 \mathrm{~kb}$ )

Additional file 8: LINC00470 promoted GBM cell proliferation. CCK8 assay was performed to determine the viability of primary GBM cells. Primary GBM cells were transfected with si-NC and si-LINC00470, pcDNA3.1, and pcDNA3.1-LINC00470, respectively. ${ }^{*} p<0.05$, ${ }^{* *} p<0.01$. (DOCX $935 \mathrm{~kb})$

\section{Abbreviations}

ANOVA: Analysis of variance; CCK-8: Cell counting kit-8; cDNA: Complementary deoxyribonucleic acid; Co-IP: Coimmunoprecipitation; GBM: Glioblastoma; GFP: Green fluorescent protein; LINC00470: Long intergenic non-protein coding RNA 470; RFP: Red fluorescent protein; RT-qPCR: Quantitative real-time polymerase chain reaction; siRNA: Small interference RNA

\section{Acknowledgements}

The authors thank Ph.D. WeiGuo Ren for his kind help in plasmid construction and Ph.D. Gang Xu and ZeYou Wang for their excellent technical assistance.

\section{Funding}

This work was supported by the National Science Foundation of China under grant number 81272297, National Key Technology Research and

Development program of the Ministry of Science and Technology of China under grant number 2014BAI04B02, 111 Project under grant number 111-2, and Graduate Research and Innovation Projects of Central South University under grant number $2017 z z t s 012$.

Availability of data and materials

Due to our internal policy, raw data cannot be shared.

\section{Authors' contributions}

$\mathrm{MW}$ and $\mathrm{CL}$ designed the study. $\mathrm{CL}, \mathrm{YZ}, \mathrm{XS}, \mathrm{PL}, \mathrm{JF}, \mathrm{HF}$, and $\mathrm{CZ}$ conducted the experiments. QL and QL acquired and managed patients and provided facilities. MW and CL wrote the article. FL revised of the manuscript. All authors read and approved the final manuscript.

\section{Ethics approval}

All of the protocols were reviewed and approved by the Joint Ethics Committee of the Central South University Health Authority and performed in accordance with national guidelines. Animal experiments were approved by the Animal Care and Use Committee of Central South University.

\section{Competing interests}

The authors declare that they have no competing interests. 


\section{Publisher's Note}

Springer Nature remains neutral with regard to jurisdictional claims in published maps and institutional affiliations.

\begin{abstract}
Author details
'Hunan Provincial Tumor Hospital and the Affiliated Tumor Hospital of Xiangya Medical School, Central South University, Changsha 410006, Hunan, China. ${ }^{2}$ Cancer Research Institute, School of Basic Medical Science, Central South University, Changsha 410078, Hunan, China. ${ }^{3}$ Key Laboratory of Carcinogenesis and Cancer Invasion, Ministry of Education, Changsha 410078, Hunan, China. ${ }^{4}$ Key Laboratory of Carcinogenesis, Ministry of Health, Changsha 410078, Hunan, China. ${ }^{5}$ Second Xiangya Hospital, Central South University, Changsha 410011, Hunan, China. 'Department of Biochemistry, University of California, Riverside, CA 92521, USA. ${ }^{7}$ Xiangya Hospital, Central South University, Changsha 410008, Hunan, China. ${ }^{8}$ Third Xiangya Hospital, Central South University, Changsha 410013, Hunan, China.
\end{abstract}

Received: 26 January 2018 Accepted: 14 May 2018

Published online: 04 June 2018

\section{References}

1. Wang RC, Wei Y, An Z, Zou Z, Xiao G, Bhagat G, et al. Akt-mediated regulation of autophagy and tumorigenesis through Beclin 1 phosphorylation. Science. 2012;338:956-9.

2. Manning BD, Toker AAKT. PKB Signaling: Navigating the Network. Cell. 2017; 169:381-405.

3. Vasudevan KM, Garraway LA. AKT signaling in physiology and disease. Curr Top Microbiol Immunol. 2010;347:105-33.

4. Massihnia D, Avan A, Funel N, Maftouh M, van Krieken A, Granchi C, et al. Phospho-Akt overexpression is prognostic and can be used to tailor the synergistic interaction of Akt inhibitors with gemcitabine in pancreatic cancer. J Hematol Oncol. 2017;10:9.

5. Zhang Y, Kwok-Shing NP, Kucherlapati M, Chen F, Liu Y, Tsang YH, et al. A Pan-Cancer Proteogenomic Atlas of PI3KJAKT/mTOR Pathway Alterations. Cancer Cell. 2017;31:820-32.

6. Fan CD, Lum MA, Xu C, Black JD, Wang X. Ubiquitin-dependent regulation of phospho-AKT dynamics by the ubiquitin E3 ligase, NEDD4-1, in the insulin-like growth factor-1 response. J Biol Chem. 2013;288:1674-84.

7. Delaloge S, DeForceville L. Targeting PI3K/AKT pathway in triple-negative breast cancer. Lancet Oncol. 2017;18:1293-4.

8. Castel P, Ellis H, Bago R, Toska E, Razavi P, Carmona FJ, et al. PDK1-SGK1 Signaling Sustains AKT-Independent mTORC1 Activation and Confers Resistance to PI3Kalpha Inhibition. Cancer Cell. 2016;30:229-42.

9. Miura H, Matsuda M, Aoki K. Development of a FRET biosensor with high specificity for Akt. Cell Struct Funct. 2014;39:9-20.

10. Gao X, Lowry PR, Zhou X, Depry C, Wei Z, Wong GW, et al. PI3K/Akt signaling requires spatial compartmentalization in plasma membrane microdomains. Proc Natl Acad Sci U S A. 2011:108:14509-14.

11. Li T, Wang G. Computer-aided targeting of the PI3K/Akt/mTOR pathway: toxicity reduction and therapeutic opportunities. Int J Mol Sci. 2014;15: 18856-91.

12. Wang R, Brattain MG. AKT can be activated in the nucleus. Cell Signal. 2006; 18:1722-31.

13. Zhan L, Wang T, Li W, Xu ZC, Sun W, Xu E. Activation of Akt/FoxO signaling pathway contributes to induction of neuroprotection against transient global cerebral ischemia by hypoxic pre-conditioning in adult rats. J Neurochem. 2010;114:897-908.

14. Farhan M, Wang H, Gaur U, Little PJ, XU J, Zheng WFOXO. Signaling Pathways as Therapeutic Targets in Cancer. Int J Biol Sci. 2017;13:815-27.

15. Gutierrez A, Look AT. NOTCH and PI3K-AKT pathways intertwined. Cancer Cell. 2007;12:411-3.

16. Itoh Y, Higuchi M, Oishi K, Kishi Y, Okazaki T, Sakai H, et al. PDK1-Akt pathway regulates radial neuronal migration and microtubules in the developing mouse neocortex. Proc Natl Acad Sci U S A. 2016;113:E2955-64.

17. Zhao L, Shan Y, Liu B, Li Y, Jia L. Functional screen analysis reveals miR-3142 as central regulator in chemoresistance and proliferation through activation of the PTEN-AKT pathway in CML. Cell Death Dis. 2017:8:e2830.

18. Fang $Y$, Xue JL, Shen Q, Chen J, Tian L. MicroRNA-7 inhibits tumor growth and metastasis by targeting the phosphoinositide 3-kinase/Akt pathway in hepatocellular carcinoma. Hepatology. 2012;55:1852-62.
19. Sun $X$, Li J, Sun Y, Zhang Y, Dong L, Shen C, et al. miR-7 reverses the resistance to BRAFi in melanoma by targeting EGFR/IGF-1R/CRAF and inhibiting the MAPK and PI3KAKT signaling pathways. Oncotarget. 2016;7: 53558-70.

20. Zhou F, Nie L, Feng D, Guo S, Luo R. MicroRNA-379 acts as a tumor suppressor in non-small cell lung cancer by targeting the IGF1R-mediated AKT and ERK pathways. Oncol Rep. 2017;38:1857-66.

21. Yang HH, Chen Y, Gao CY, Cui ZT, Yao JM. Protective Effects of MicroRNA126 on Human Cardiac Microvascular Endothelial Cells Against Hypoxia/ Reoxygenation-Induced Injury and Inflammatory Response by Activating PI3K/Akt/eNOS Signaling Pathway. Cell Physiol Biochem. 2017:42:506-18.

22. Lin A, Hu Q, Li C, Xing Z, Ma G, Wang C, et al. The LINK-A IncRNA interacts with Ptdlns $(3,4,5) P 3$ to hyperactivate AKT and confer resistance to AKT inhibitors. Nat Cell Biol. 2017;19:238-51.

23. Yang $N$, Chen J, Zhang $H$, Wang $X$, Yao H, Peng $Y$, et al. LncRNA OIP5-AS1 loss-induced microRNA-410 accumulation regulates cell proliferation and apoptosis by targeting KLF10 via activating PTEN/PI3K/KT pathway in multiple myeloma. Cell Death Dis. 2017;8:e2975.

24. Jin Y, Feng SJ, Qiu S, Shao N, Zheng JH. LncRNA MALAT1 promotes proliferation and metastasis in epithelial ovarian cancer via the PI3K-AKT pathway. Eur Rev Med Pharmacol Sci. 2017;21:3176-84

25. Xing Z, Lin A, Li C, Liang K, Wang S, Liu Y, et al. IncRNA directs cooperative epigenetic regulation downstream of chemokine signals. Cell. 2014;159:1110-25.

26. Chen LL. Linking Long Noncoding RNA Localization and Function. Trends Biochem Sci. 2016:41:761-72.

27. Quinn JJ, Chang HY. Unique features of long non-coding RNA biogenesis and function. Nat Rev Genet. 2016;17:47-62.

28. Rinn JL, Chang HY. Genome regulation by long noncoding RNAs. Annu Rev Biochem. 2012;81:145-66.

29. Goff LA, Rinn JL. Linking RNA biology to IncRNAs. Genome Res. 2015;25: 1456-65.

30. Wang KC, Yang YW, Liu B, Sanyal A, Corces-Zimmerman $R$, Chen $Y$, et al. A long noncoding RNA maintains active chromatin to coordinate homeotic gene expression. Nature. 2011;472:120-4.

31. Flynn RA, Chang HY. Long noncoding RNAs in cell-fate programming and reprogramming. Cell Stem Cell. 2014;14:752-61.

32. Wu H, Yin QF, Luo Z, Yao RW, Zheng CC, Zhang J, et al. Unusual Processing Generates SPA LncRNAs that Sequester Multiple RNA Binding Proteins. Mol Cell. 2016;64:534-48.

33. Stohr H, Mah N, Schulz HL, Gehrig A, Frohlich S, Weber BH. EST mining of the UniGene dataset to identify retina-specific genes. Cytogenet Cell Genet. 2000;91:267-77.

34. Ota T, Suzuki Y, Nishikawa T, Otsuki T, Sugiyama T, Irie R, et al. Complete sequencing and characterization of 21,243 full-length human cDNAs. Nat Genet. 2004;36:40-5.

35. Liu C, Sun Y, She $X$, Tu C, Cheng X, Wang L, et al. CASC2C as an unfavorable prognosis factor interacts with miR-101 to mediate astrocytoma tumorigenesis. Cell Death Dis. 2017:8:e2639.

36. Yu Z, Sun $Y$, She $X$, Wang $Z$, Chen $S$, Deng $Z$, et al. SIX3, a tumor suppressor, inhibits astrocytoma tumorigenesis by transcriptional repression of AURKA B. J Hematol Oncol. 2017:10:115.

37. Xiaoping L, Zhibin Y, Wenjuan L, Zeyou W, Gang X, Zhaohui L, et al. CPEB1, a histone-modified hypomethylated gene, is regulated by miR-101 and involved in cell senescence in glioma. Cell Death Dis. 2013;4:e675.

38. Nakaya T, Alexiou P, Maragkakis M, Chang A, Mourelatos Z. FUS regulates genes coding for RNA-binding proteins in neurons by binding to their highly conserved introns. Rna. 2013;19:498-509.

39. Kovar H. Dr. Jekyll and Mr. Hyde: The Two Faces of the FUS/EWS/TAF15 Protein Family. Sarcoma. 2011;2011:837474.

40. Lagier-Tourenne C, Polymenidou M, Hutt KR, Vu AQ, Baughn M, Huelga SC, et al. Divergent roles of ALS-linked proteins FUS/TLS and TDP-43 intersect in processing long pre-mRNAs. Nat Neurosci. 2012;15:1488-97.

41. Le Grand M, Berges R, Pasquier E, Montero MP, Borge L, Carrier A, et al. Akt targeting as a strategy to boost chemotherapy efficacy in non-small cell lung cancer through metabolism suppression. Sci Rep. 2017;7:45136.

42. Han F, Xiao QQ, Peng S, Che XY, Jiang LS, Shao Q, et al. Atorvastatin ameliorates LPS-induced inflammatory response by autophagy via AKT/ mTOR signaling pathway. J Cell Biochem. 2018;119:1604-15.

43. Mo Q, Hu L, Weng J, Zhang Y, Zhou Y, Xu R, et al. Euptox A Induces G1 Arrest and Autophagy via p38 MAPK- and PI3K/Akt/mTOR-Mediated Pathways in Mouse Splenocytes. J Histochem Cytochem. 2017;65:543-58. 
44. Liu C, Liu Z, Li X, Tang X, He J, Lu S. MicroRNA-1297 contributes to tumor growth of human breast cancer by targeting PTEN/PI3K/AKT signaling. Oncol Rep. 2017;38:2435-43.

45. Smith TA. Mammalian hexokinases and their abnormal expression in cancer. Br J Biomed Sci. 2000;57:170-8.

46. Yoshida GJ. Therapeutic strategies of drug repositioning targeting autophagy to induce cancer cell death: from pathophysiology to treatment. J Hematol Oncol. 2017;10:67.

47. Fulda S. Targeting autophagy for the treatment of cancer. Biol Chem. 2018; [Epub ahead of print]

48. Liu X, Chong Y, Tu Y, Liu N, Yue C, Qi Z, et al. CRM1/XPO1 is associated with clinical outcome in glioma and represents a therapeutic target by perturbing multiple core pathways. J Hematol Oncol. 2016;9:108.

49. Akinleye A, Avvaru P, Furqan M, Song Y, Liu D. Phosphatidylinositol 3-kinase (PI3K) inhibitors as cancer therapeutics. J Hematol Oncol. 2013;6:88.

50. Liu D, Zhu Y, Pang J, Weng X, Feng X, Guo Y. Knockdown of long noncoding RNA MALAT1 inhibits growth and motility of human hepatoma cells via modulation of miR-195. J Cell Biochem. 2018;119:1368-80.

51. Chen SW, Zhu J, Ma J, Zhang JL, Zuo S, Chen GW, et al. Overexpression of long non-coding RNA H19 is associated with unfavorable prognosis in patients with colorectal cancer and increased proliferation and migration in colon cancer cells. Oncol Lett. 2017:14:2446-52.

52. Zinszner H, Sok J, Immanuel D, Yin Y, Ron D. TLS (FUS) binds RNA in vivo and engages in nucleo-cytoplasmic shuttling. J Cell Sci. 1997;110(Pt 15): $1741-50$.

53. Tan AY, Manley JL. TLS/FUS: a protein in cancer and ALS. Cell Cycle. 2012;11: 3349-50.

\section{Ready to submit your research? Choose BMC and benefit from:}

- fast, convenient online submission

- thorough peer review by experienced researchers in your field

- rapid publication on acceptance

- support for research data, including large and complex data types

- gold Open Access which fosters wider collaboration and increased citations

- maximum visibility for your research: over $100 \mathrm{M}$ website views per year

At BMC, research is always in progress.

Learn more biomedcentral.com/submissions 